\title{
Article
}

\author{
Doi 10.5943/mycosphere/8/8/14
}

Copyright $($ ) Guizhou Academy of Agricultural Sciences

\section{New Cylindrocladiella spp. from Thailand soils}

\section{Lombard $\mathbf{L}^{1^{*}}$, Cheewangkoon $\mathbf{R}^{2^{*}}$, Crous $\mathbf{P W}^{1,2}$}

\author{
${ }^{1}$ Westerdijk Fungal Biodiversity Institute, Uppsalalaan 8, 3584 CT Utrecht, The Netherlands \\ ${ }^{2}$ Department of Entomology and Plant Pathology, Faculty of Agriculture, Chiang Mai University, Chiang Mai 50200, \\ Thailand
}

Lombard L, Cheewangkoon R, Crous PW 2017 - New Cylindrocladiella spp. from Thailand soils. Mycosphere 8(8), 1088-1104, Doi 10.5943/mycosphere/8/8/14

\begin{abstract}
Species of Cylindrocladiella (Nectriaceae) are generally regarded as saprobes or weak plant pathogens, typically causing problems in nurseries under high humidity conditions. Although the genus Cylindrocladiella is a common soil inhabitant in tropical and subtropical countries, only seven species have thus far been reported from Thailand. The aim of this study was thus to investigate various soil samples from northern Thailand for the presence of Cylindrocladiella, and to identify isolates to species level using a combination of cultural studies, morphology and DNA phylogeny (ITS, tef1 and tub2 gene regions). From the various isolates obtained, six new Cylindrocladiella species could be identified, described here as C. brevistipitata, C. horticola, C. humicola, $C$. lateralis, $C$. reginae, and $C$. terrestris. Further studies are required to establish the ecology of the new species, and also to determine which potential species occur in other as yet unsampled regions in Thailand.
\end{abstract}

Key words - cryptic species - phylogeny - taxonomy

\section{Introduction}

Boesewinkel (1982) established the asexual genus Cylindrocladiella, based on C. parva, to accommodate several cylindrocladium-like (=Calonectria) species characterised by small $(<20 \mu \mathrm{m}$ long), 1-septate, cylindrical conidia and aseptate, thick-walled stipe extensions. Initially, Cylindrocladiella spp. were linked to the sexual genus Nectricladiella (Crous \& Wingfield 1993, Schoch et al. 2000), however, following the implementation of the International Code of Nomenclature for algae, fungi, and plants (ICN, McNeill et al. 2012), Rossman et al. (2013) proposed that the genus name Cylindrocladiella be retained over Nectricladiella. Recently, Lombard et al. (2015) showed that the genus Cylindrocladiella formed a monophyletic group in the Nectriaceae, being closely related to genera such as Aquanectria and Gliocladiopsis.

To date, 29 species of Cylindrocladiella are recognized (Crous \& Wingfield 1993, Coller et al. 2005, Inderbitzin et al. 2012, Lombard et al. 2012, 2015, Crous et al. 2017), of which two are linked to nectricladiella-like sexual morphs (Schoch et al. 2000, Crous 2002, Lombard et al. 2012, 2015). These fungi are generally soil-borne, and regarded as saprobes or weak plant pathogens with wide host ranges (Crous 2002, van Coller et al. 2005, Scattolin \& Montecchio 2007, Lombard et al. 2012). Disease symptoms associated with Cylindrocladiella infection include leaf spots and root, 
stem and cutting rots (Crous et al. 1991, Peerally 1991, Crous \& Wingfield 1993, Crous 2002, van Coller et al. 2005, Scattolin \& Montecchio 2007, Lombard et al. 2012).

The aim of this study was to consider the identity of several Cylindrocladiella isolates isolated from soils collected in Thailand. To achieve this goal, combined morphological characteristics and phylogenetic inference was employed to identify these Cylindrocladiella isolates to species level.

\section{Materials \& Methods}

\section{Isolates}

Soils were collected from various regions in Thailand, and baited as described by Crous (2002) using sterilised alfalfa seeds (Table 1). Representative strains are maintained in the culture collection of the Westerdijk Fungal Biodiversity Institute (CBS).

\section{Phylogeny}

Total genomic DNA was extracted from axenic cultures grown on 2\% malt extract agar (MEA) for 7 days, using the Wizard ${ }^{\circ}$ Genomic DNA Purification Kit (Promega Corporation, Madison, WI) according to the manufacturer's protocol. Partial gene sequences were determined for $\beta$-tubulin (tub2), the internal transcribed spacer region (ITS) of the nuclear rDNA and translation elongation factor 1-alpha (tefl) using the primers and protocols described previously (Lombard et al. 2012, 2015). Subsequent alignments were generated using MAFFT v. 7 (Katoh \& Standley 2013), and manually corrected where necessary.

The sequence datasets were tested for congruency using the reciprocal $70 \%$ bootstrap (BS) threshold method as described by Gueidan et al. (2007) to determine if the three partitions could be combined. Phylogenetic analyses were based on Bayesian inference (BI), maximum parsimony (MP) and maximum likelihood (ML). For BI and ML, the best evolutionary models for each partition were determined using MrModeltest (Nylander 2004) and incorporated into the analysis. MrBayes v. 3.2.1. (Ronquist \& Huelsenbeck 2003) was used to generate phylogenetic trees under optimal criteria per partition. A Markov Chain Monte Carlo (MCMC) algorithm of four chains was started in parallel from a random tree topology with the heating parameter set at 0.3. The MCMC analysis lasted until the average standard deviation of split frequencies came below 0.01 with trees saved each 1000 generations. The first $25 \%$ of saved trees were discarded as the "burn-in" phase and posterior probabilities (PP) determined from the remaining trees.

The MP analysis was done using PAUP (Phylogenetic Analysis Using Parsimony, v. 4.0b10, Swofford 2002). Phylogenetic relationships were estimated by heuristic searches with 1000 random addition sequences. Tree bisection-reconnection was used, with the branch swapping option set on 'best trees' only with all characters weighted equally and alignment gaps treated as fifth state. Tree length (TL), consistency index (CI), retention index (RI) and rescaled consistence index (RC) were calculated for parsimony and bootstrap analysis (Hillis \& Bull 1993) was based on 1000 replications.

The ML analysis was made with RAxML (randomized axelerated [sic] maximum likelihood for high performance computing; Stamatakis 2014) through the CIPRES website (http://www.phylo.org) to obtain a second measure of branch support. The robustness of the analysis was evaluated by bootstrap support (BS) analysis with the bootstrap replicates automatically determined by the software. Novel sequences were deposited in GenBank (Table 1) and all alignments in TreeBase as $\underline{\mathrm{S} 21153}$.

\section{Taxonomy}

Axenic cultures were grown on synthetic nutrient-poor agar (SNA; Nirenburg 1981) amended with sterile carnation leaf pieces, and on MEA as described by Lombard et al. (2012). Gross morphological characteristics were studied by mounting the fungal structures in $85 \%$ lactic acid and 30 measurements were made for all taxonomically informative characters at $\times 1000$ magnification using a Plan-Apochromat $\times$ 100/1.4 oil immersion lens (Carl Zeiss, Germany) mounted on a Zeiss 
Axioscope 2 microscope, with differential interference contrast (DIC) illumination. The $95 \%$ confidence levels were determined for the conidial measurements with extremes given in parentheses. For all other fungal structures measured, only the extremes are provided. Colony colour was assessed using 7-day-old cultures on MEA incubated at room temperature and the colour charts of Rayner (1970). All descriptions, illustrations and nomenclatural data were deposited in MycoBank (Crous et al. 2004).

\section{Results}

\section{Phylogeny}

Approximately 500-550 bases were determined for the three loci used in this study. The $70 \%$ reciprocal bootstrap tree topologies for the three loci revealed a conflict between the coding gene regions (tefl \& tub2) and the non-coding ITS gene region. The ITS gene region was unable to resolve majority of the established Cylindrocladiella spp. However, the three loci were still combined into a single dataset based on the argument of Cunningham (1997) that combining incongruent partitions could increase phylogenetic accuracy.

The combined alignment of the ITS, tefl and tub2 contained 1579 characters from 68 taxa, including Gliocladiopsis sagariensis (CBS 199.55; Lombard et al. 2015) as the outgroup. The number of unique site patterns per data partition, including alignment gaps, was 61 from 503 characters for ITS, 203 from 547 characters for tefl, and 176 from 528 characters for tub2. MrModeltest determined that all three partitions had dirichlet base frequencies. A GTR+I+G model with inverse gamma-distributed rates was used for ITS and tefl, while $\mathrm{HKY}+\mathrm{I}+\mathrm{G}$ with inverse gamma-distributed rates was implemented for tub2.

The Bayesian analysis lasted 15152 generations and the consensus tree, with posterior probabilities, was calculated from 7576 trees left after 2525 trees were discarded as burn-in. For the MP analysis 1058 characters were constant, 175 parsimony-uninformative, and 346 parsimonyinformative, yielding 22 equally most parsimonious trees $(\mathrm{TL}=1260 ; \mathrm{CI}=0.571$; RI $=0.886$; RC $=0.505)$. ML analysis resulted in a single best ML tree with $-\mathrm{InL}=-7042.460298$. The best ML tree confirmed the consensus tree topologies obtained from the BI and MP analyses, and therefore only the best ML tree is presented (Fig. 1).

In the phylogenetic tree (Fig. 1), the majority of the Cylindrocladiella isolates obtained from soils collected in Thailand clustered into five highly-supported clades, with isolate CBS 142786 forming a distinct single lineage closely related to $C$. infestans and isolates CBS 142787 \& CBS 142788. Both later isolates formed a distinct clade (ML-BS \& MP-BS > 75 \%; PP =1.0) from the C. infestans clade (CBS 191.50 \& CBS 111795), representing a novel phylogenetic species. Isolates CBS 142777, CBS 142778 and CBS 142779 formed a distinct clade (ML-BS \& MP-BS > 75 \%; PP $=1.0$ ) closely related to $C$. clavata (CBS $129563 \&$ CBS 129564), whereas CBS 142780, CBS 142781, CBS 142782 and CBS 142783 formed a unique clade (ML-BS \& MP-BS > $75 \%$; PP = 1.0), distantly related to $C$. parva (CBS 114524), C. pseudoparva (CBS 113624 \& CBS 129560) and $C$. stellenboschensis (CBS 110688 \& CBS 115611). Isolates CBS 142784 and CBS 142785 also formed a unique clade (ML-BS \& MP-BS $=100 \%$; PP =1.0), distinct from $C$. kurandica (CBS 129576 \& CBS 129577), C. pseudoinfestans (CBS 114530 \& CBS 114531) and C. queenslandica (CBS 129574 \& CBS 129575), representing a novel phylogenetic species. Isolates CBS 142789 and CBS 142790 formed a distinct clade (ML-BS \& MP-BS $=100 \%$; PP $=1.0$ ), sister to C. peruviana (CBS 114697 \& IMUR 1843).

\section{Taxonomy}

Based on phylogenetic inference and morphological observations, six novel Cylindrocladiella species could be identified, isolated from the soils collected in Thailand, and are, therefore, described below. 
Table 1. Isolates of Cylindrocladiella included in the study.

\begin{tabular}{|c|c|c|c|c|c|c|c|}
\hline \multirow[t]{2}{*}{ Species } & \multirow[t]{2}{*}{ Isolates } & \multicolumn{3}{|c|}{ GenBank Accessions } & \multirow[t]{2}{*}{ Substrate } & \multirow[t]{2}{*}{ Country } & \multirow[t]{2}{*}{ Collector } \\
\hline & & ITS & tef1 & tub2 & & & \\
\hline \multirow[t]{2}{*}{ C. australiensis } & CBS $129567=$ CPC $17507^{\mathrm{T}}$ & JN100624 & JN099060 & JN098747 & Soil & Australia & P.W. Crous \\
\hline & CBS $129568=$ CPC 17562 & JN100623 & JN099059 & JN098748 & Soil & Australia & P.W. Crous \\
\hline \multirow[t]{2}{*}{ C. camelliae } & CPC234 = PPRI 3990 & AF220952 & JN099087 & AY793471 & E. grandis & South Africa & P.W. Crous \\
\hline & CBS $114891=$ CPC 277 & AF220953 & JN099086 & AY793472 & E. grandis & South Africa & P.W. Crous \\
\hline C. brevistipitata & CBS $142786^{\mathrm{T}}$ & - & MF444940 & MF444926 & Soil & Thailand & P.W. Crous \\
\hline \multirow[t]{2}{*}{ C. clavata } & CBS $129563=$ CPC 17591 & JN099096 & JN098975 & JN098751 & Soil & Australia & P.W. Crous \\
\hline & CBS $129564=$ CPC $17592^{\mathrm{T}}$ & JN099095 & JN098974 & JN098752 & Soil & Australia & P.W. Crous \\
\hline C. cymbiformis & CBS $129553=$ CPC $17393^{T}$ & JN099103 & JN098988 & JN098753 & Soil & Australia & P.W. Crous \\
\hline \multirow[t]{2}{*}{ C. elegans } & CBS $338.92=$ IMI $346847^{\mathrm{T}}$ & AY793444 & JN099039 & AY793474 & Leaf litter & South Africa & I. Rong \\
\hline & CBS $110801=$ CPC 525 & JN100609 & JN099044 & JN098755 & Leaf litter & South Africa & P.W. Crous \\
\hline \multirow[t]{2}{*}{ C. ellipsoidea } & CBS $129572=$ CPC 17558 & JN100636 & JN099073 & JN098756 & Soil & Australia & P.W. Crous \\
\hline & CBS $129573=$ CPC $17560^{\mathrm{T}}$ & JN099094 & JN098973 & JN098757 & Soil & Australia & P.W. Crous \\
\hline C. hahajimaensis & PD684 & JN687561 & JN687562 & - & Soil & Japan & T. Watanabe \\
\hline \multirow[t]{2}{*}{ C. hawaiiensis } & CBS 118704 & JN099115 & JN098996 & JN098760 & Soil & Hawaii & Y. Degawa \\
\hline & CBS $129569=\mathrm{CPC} 12272^{\mathrm{T}}$ & JN100621 & JN099057 & JN098761 & Soil & Hawaii & Y. Degawa \\
\hline \multirow[t]{2}{*}{ C. horticola } & CBS $142784^{\mathrm{T}}$ & MF444911 & MF444938 & MF444924 & Soil & Thailand & P.W. Crous \\
\hline & CBS 142785 & MF444912 & MF444939 & MF444925 & Soil & Thailand & P.W. Crous \\
\hline \multirow[t]{3}{*}{ C. humicola } & CBS 142777 & MF444904 & MF444931 & MF444917 & Soil & Thailand & P.W. Crous \\
\hline & CBS 142778 & MF444905 & MF444932 & MF444918 & Soil & Thailand & P.W. Crous \\
\hline & CBS $142779^{\mathrm{T}}$ & MF444906 & MF444933 & MF444919 & Soil & Thailand & P.W. Crous \\
\hline \multirow[t]{2}{*}{ C. infestans } & CBS $111795=$ ATCC $44816^{\mathrm{T}}$ & AF220955 & JN099037 & AF320190 & Pinus pinea & New Zealand & H.J. Boesewinkel \\
\hline & CBS $191.50=$ IMI 299376 & AF220956 & JN099036 & AY793475 & Arenga pinnata & Indonesia & $\begin{array}{l}\text { K.B. Boedijn \& J. } \\
\text { Reitsma }\end{array}$ \\
\hline \multirow[t]{2}{*}{ C. kurandica } & CBS $129576=$ CPC 17547 & JN100634 & JN099071 & JN098764 & Soil & Australia & P.W. Crous \\
\hline & CBS $129577=$ CPC $17551^{\mathrm{T}}$ & JN100646 & JN099083 & JN098765 & Soil & Australia & P.W. Crous \\
\hline
\end{tabular}




\begin{tabular}{|c|c|c|c|c|c|c|c|}
\hline \multirow[t]{2}{*}{ C. lageniformis } & CBS $340.92=\mathrm{UFV} 115^{\mathrm{T}}$ & AF220959 & JN099003 & AY793481 & Eucalyptus sp. & Brazil & A.C. Alfenas \\
\hline & CBS $111060=$ CPC 1240 & JN100611 & JN099046 & JN098770 & Eucalyptus sp. & South Africa & P.W. Crous \\
\hline \multirow[t]{2}{*}{ C. lanceolata } & CBS $129565=$ CPC 17566 & JN100632 & JN099069 & JN098788 & Soil & Australia & P.W. Crous \\
\hline & CBS $129566=\mathrm{CPC} 17567^{\mathrm{T}}$ & JN099099 & JN098978 & JN098789 & Soil & Australia & P.W. Crous \\
\hline \multirow[t]{2}{*}{ C. lateralis } & CBS 142787 & MF444913 & MF444941 & MF444927 & Soil & Thailand & P.W. Crous \\
\hline & CBS $142788^{\mathrm{T}}$ & MF444914 & MF444942 & MF444928 & Soil & Thailand & P.W. Crous \\
\hline \multirow[t]{2}{*}{ C. longiphialidica } & CBS $129557=\mathrm{CPC} 18839^{\mathrm{T}}$ & JN100585 & JN098966 & JN098790 & Soil & Thailand & P.W. Crous \\
\hline & CBS $129558=$ CPC 18841 & JN100586 & JN098967 & JN098791 & Soil & Thailand & P.W. Crous \\
\hline \multirow[t]{2}{*}{ C. longistipitata } & CBS $112953=$ CPC 4720 & JN100595 & JN099025 & JN098792 & O. heterophylla & Australia & $\begin{array}{l}\text { C. Pearce \& B. } \\
\text { Paulus }\end{array}$ \\
\hline & CBS $116075=\mathrm{CPC} 708^{\mathrm{T}}$ & AF220958 & JN098993 & AY793506 & Soil & China & M.J. Wingfield \\
\hline C. microcylindrica & CBS $111794=\operatorname{ATCC} 38571^{\mathrm{T}}$ & AY793452 & JN099041 & AY793483 & E. elegans & Indonesia & C.F. Hill \\
\hline \multirow[t]{2}{*}{ C. natalensis } & CBS $114943=\mathrm{CPC} 456^{\mathrm{T}}$ & JN100588 & JN099016 & JN098794 & A. hypogaea & South Africa & M.J. Wingfield \\
\hline & CBS $114944=$ CPC 457 & JN100589 & JN099017 & JN098795 & A. hypogaea & South Africa & M.J. Wingfield \\
\hline \multirow[t]{2}{*}{ C. nederlandica } & CBS $146.94=$ PD39/1776 & JN099127 & JN099011 & JN098799 & $\begin{array}{l}\text { Rhododendron } \\
\text { sp. }\end{array}$ & The Netherlands & \\
\hline & CBS $152.91=\mathrm{PD} 90 / 2015^{\mathrm{T}}$ & JN100603 & JN099033 & JN098800 & Pelargonium sp. & The Netherlands & $\begin{array}{l}\text { J.W. Veenbaas- } \\
\text { Rijks }\end{array}$ \\
\hline C. novaezelandica & CBS $486.77=$ ATCC $44815^{\mathrm{T}}$ & AF220963 & JN099050 & AY793485 & $\begin{array}{l}\text { Rhododendron } \\
\text { indicum }\end{array}$ & New Zealand & H.J. Boesewinkel \\
\hline C. parva & CBS $114524=$ ATCC $28272^{\mathrm{T}}$ & AF220964 & JN099009 & AY793486 & $\begin{array}{l}\text { Telopea } \\
\text { speciosissima }\end{array}$ & New Zealand & H.J. Boesewinkel \\
\hline \multirow[t]{2}{*}{ C. peruviana } & CBS $114697=$ CPC 2573 & JN099124 & JN099007 & JN098802 & Vitis vinifera & South Africa & S. Lambrecht \\
\hline & IMUR $1843=\mathrm{CPC} 2404^{\mathrm{T}}$ & AF220966 & JN098968 & AY793500 & ants & Peru & M.P. Herrera \\
\hline \multirow[t]{2}{*}{ C. pseudocamelliae } & CBS $129555=\mathrm{CPC} 18825^{\mathrm{T}}$ & JN100577 & JN098958 & JN098814 & Soil & Thailand & P.W. Crous \\
\hline & CBS $129556=$ CPC 18832 & JN100580 & JN098961 & JN098815 & Soil & Thailand & P.W. Crous \\
\hline \multirow{2}{*}{$\begin{array}{l}\text { C. } \\
\text { pseudohawaiiensis }\end{array}$} & CBS $210.94=$ PPRI $4450^{\mathrm{T}}$ & JN099128 & JN099012 & JN098819 & Eucalyptus sp. & Brazil & A.C. Alfenas \\
\hline & $\mathrm{CBS} 115610=\mathrm{CPC} 909$ & N100594 & JN099024 & JN098820 & & gascar & Crous \\
\hline C. pseudoinfestans & CBS $114530=$ CPC 2320 & JN099126 & JN099010 & JN098821 & Soil & Madagascar & J.E. Taylor \\
\hline
\end{tabular}




\begin{tabular}{|c|c|c|c|c|c|c|c|}
\hline & $\operatorname{CBS} 114531=\operatorname{CPC} 2319^{\mathrm{T}}$ & AF220957 & JN099004 & AY793508 & Soil & Madagascar & J.E. Taylor \\
\hline \multirow[t]{2}{*}{ C. pseudoparva } & CBS $113624=$ CPC 752 & JN099121 & JN099002 & JN098822 & Quercus sp. & Switzerland & L. Petrini \\
\hline & CBS $129560=$ CPC $18149^{\mathrm{T}}$ & JN100620 & JN099056 & JN098824 & Soil & The Netherlands & P.W. Crous \\
\hline \multirow[t]{2}{*}{ C. queenslandica } & CBS $129574=$ CPC $17568^{\mathrm{T}}$ & JN099098 & JN098977 & JN098826 & Soil & Australia & P.W. Crous \\
\hline & CBS $129575=$ CPC 17569 & JN099097 & JN098976 & JN098827 & Soil & Australia & P.W. Crous \\
\hline \multirow[t]{4}{*}{ C. reginae } & CBS 142780 & MF444907 & MF444934 & MF444920 & Soil & Thailand & P.W. Crous \\
\hline & CBS 142781 & MF444908 & MF444935 & MF444921 & Soil & Thailand & P.W. Crous \\
\hline & CBS $142782^{\mathrm{T}}$ & MF444909 & MF444936 & MF444922 & Soil & Thailand & P.W. Crous \\
\hline & CBS 142783 & MF444910 & MF444937 & MF444923 & Soil & Thailand & P.W. Crous \\
\hline \multirow[t]{2}{*}{ C. queenslandica } & CBS $129574=$ CPC $17568^{\mathrm{T}}$ & JN099098 & JN098977 & JN098826 & soil & Australia & P.W. Crous \\
\hline & CBS $129575=$ CPC 17569 & JN099097 & JN098976 & JN098827 & soil & Australia & P.W. Crous \\
\hline \multirow[t]{2}{*}{ C. stellenboschensis } & $\mathrm{CBS} 110668=\mathrm{CPC} 517^{\mathrm{T}}$ & JN100615 & JN099051 & JN098829 & soil & South Africa & P.W. Crous \\
\hline & CBS $115611=$ CPC 4074 & JN100593 & JN099023 & JN098830 & Geum sp. & New Zealand & P.W. Crous \\
\hline \multirow[t]{2}{*}{ C. terrestris } & CBS 142789 & MF444915 & MF444943 & MF444929 & Soil & Thailand & P.W. Crous \\
\hline & CBS 142790 & MF444916 & MF444944 & MF444930 & Soil & Thailand & P.W. Crous \\
\hline \multirow[t]{2}{*}{ C. thailandica } & CBS $129570=$ CPC 18834 & JN100581 & JN098962 & JN098833 & soil & Thailand & P.W. Crous \\
\hline & $\mathrm{CBS} 129571=\mathrm{CPC} 18835^{\mathrm{T}}$ & $\mathrm{JN} 100582$ & JN098963 & JN098834 & soil & Thailand & P.W. Crous \\
\hline \multirow[t]{2}{*}{ C. variabilis } & CBS $375.93=$ IMI 317057 & JN099119 & JN099000 & JN098836 & Mangifera indica & India & P.N. Chowdhry \\
\hline & CBS $129561=\mathrm{CPC} 17505^{\mathrm{T}}$ & JN100643 & JN099080 & JN098719 & soil & Australia & P.W. Crous \\
\hline \multirow[t]{2}{*}{ C. viticola } & CBS $112897=$ CPC $5606^{\mathrm{T}}$ & AY793468 & JN099064 & AY793504 & Vitis vinifera & South Africa & G.J. van Coller \\
\hline & CBS $114682=$ IMI 297470 & JN100612 & JN099047 & JN098723 & $\begin{array}{l}\text { Amorphophallus } \\
\text { sp. }\end{array}$ & Thailand & R. Stevenson \\
\hline
\end{tabular}

CBS: Westerdijk Fungal Biodiversity Institute (WI), Utrecht, The Netherlands; CPC: working collection of Pedro Crous housed at WI; IMI: International Mycological Institute, CABI-Bioscience, Egham, Bakeham Lane, U.K.; ATCC: American Type Culture Collection, Virginia, U.S.A.; PPRI: Plant Protection Research Institute, Agricultural Research Council, Pretoria, South Africa.; UFV: Univeridade Federal de Vicosa, Brazil. ITS = Internal transcribed spacer regions 1 and 2 and the 5.8S gene of the ribosomal RNA; tef1 = Translation elongation factor 1 -alpha; tub2 $=\beta$-tubulin. ${ }^{\mathrm{T}}$ Ex-type cultures 


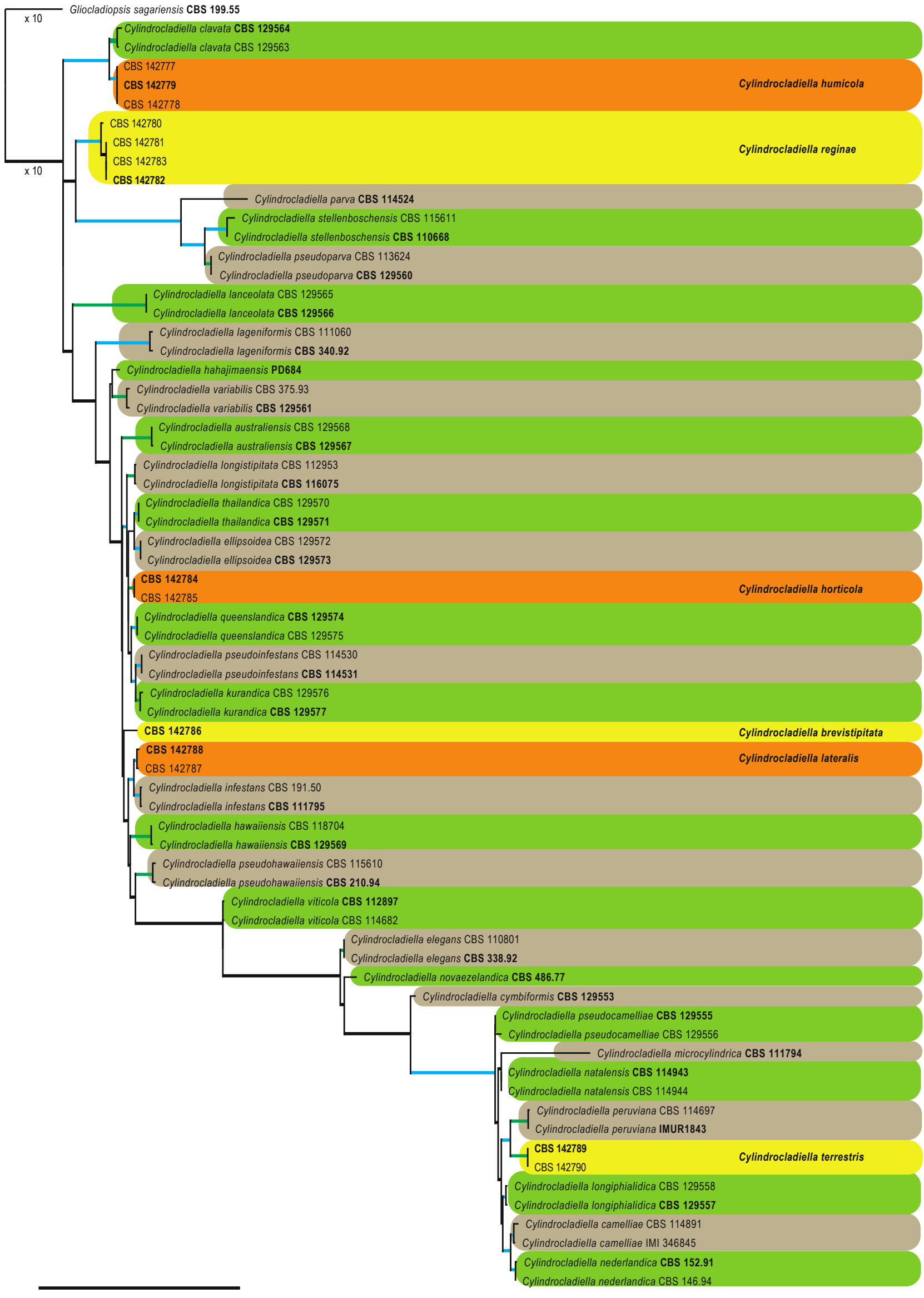


Figure 1 - The ML consensus tree inferred from the combined ITS, tef1 and tub2 sequence alignments. Thickened branches indicate branches present in the ML, MP and Bayesian consensus trees. Branches with ML-BS \& MP-BS $=100 \%$ and PP $=1.0$ are in green. Branches with ML-BS $\&$ MP-BS $\geq 75 \%$ and $\mathrm{PP} \geq 0.95$ are in blue. The scale bar indicates 0.2 expected changes per site. The tree is rooted to Gliocladiopsis sagariensis (CBS 199.55). Epi- and ex-type strains are indicated in bold.

Cylindrocladiella brevistipitata L. Lombard \& Crous, sp. nov.

Fig. 2 MycoBank MB821579; Facesoffungi number: 03582

Etymology - Name refers to the short stipe extension of the conidiophores.

Sexual morph unknown. Conidiophores penicillate, mononematous and hyaline, comprising a stipe, a penicillate arrangement of fertile branches, a stipe extension and a terminal vesicle; stipe septate, hyaline, smooth, 75-150 × 4-6 $\mu \mathrm{m}$; stipe extension aseptate, straight, $60-95 \mu \mathrm{m}$ long, thickwalled with one basal septum, terminating in thin-walled, ellipsoidal to lanceolate vesicles, $4-5 \mu \mathrm{m}$ wide. Penicillate conidiogenous apparatus with primary branches aseptate, 10-26 $\times 2-4 \mu \mathrm{m}$, secondary branches aseptate, $8-15 \times 2-4 \mu \mathrm{m}$, tertiary branches aseptate, 6-11 $\times 2-3 \mu \mathrm{m}$, each terminal branch producing 2-4 phialides; phialides cymbiform to cylindrical, hyaline, aseptate, 8$14 \times 2-3 \mu \mathrm{m}$, apex with minute periclinal thickening and collarette. Subverticillate conidiophores not observed. Conidia cylindrical, rounded at both ends, straight, 1-septate, (9-)10-12 × 2-3 $\mu \mathrm{m}$ $($ av. $=11 \times 2 \mu \mathrm{m})$, frequently slightly flattened at the base, held in asymmetrical clusters by colourless slime.

Culture characteristics - Colonies convex, cottony, with sinuate margins, honey with umber centre; reverse sienna to umber; chlamydospores extensive throughout medium arranged in chains; reaching $65-75 \mathrm{~mm}$ after $7 \mathrm{~d}$ on MEA at $24{ }^{\circ} \mathrm{C}$ in ambient light.

Material examined - Thailand, Mae Rim, Queen Sirikit Botanical Garden, from soil, October 2010, P.W. Crous (holotype CBS H-23173, culture ex-type CBS 142786).

Notes - Cylindrocladiella brevistipitata can be distinguished from other species in the genus by its relatively short stipe extensions, and tertiary branches formed on its penicillate conidiogenous apparatus.

Cylindrocladiella horticola L. Lombard \& Crous, sp. nov.

Fig. 3

MycoBank MB821580; Facesoffungi number: FoF03583

Etymology - Named refers to the locality, Queen Sirikit Botanical Garden, from where this fungus was collected.

Sexual morph unknown. Conidiophores dimorphic, penicillate and subverticillate, mononematous and hyaline, comprising a stipe, a penicillate arrangement of fertile branches, a stipe extension and a terminal vesicle; stipe septate, hyaline, smooth, 50-100 $\times 4-7 \mu \mathrm{m}$; stipe extension aseptate, straight, 55-150 $\mu \mathrm{m}$ long, thick-walled with one basal septum, terminating in thin-walled, ellipsoidal to lanceolate vesicles, 3-6 $\mu \mathrm{m}$ wide. Penicillate conidiogenous apparatus with primary branches aseptate, 10-20 × 2-4 $\mu \mathrm{m}$, secondary branches aseptate, 7-12 $\times 2-4 \mu \mathrm{m}$, each terminal branch producing 2-4 phialides; phialides doliiform to reniform to cymbiform, hyaline, aseptate, 7$14 \times 2-3 \mu \mathrm{m}$, apex with minute periclinal thickening and collarette. Subverticillate conidiophores abundant, comprising of a septate stipe and primary branches terminating in 2-4 phialides; primary branches straight, hyaline, 0-1-septate, 15-40 × 2-4 $\mu \mathrm{m}$; phialides cymbiform to cylindrical, hyaline, aseptate, $11-18 \times 2-3 \mu \mathrm{m}$, apex with minute periclinal thickening and collarette. Conidia cylindrical, rounded at both ends, straight, 1-septate, $(11-) 11.5-12.5(-14) \times(1-) 1.5-2.5(-3) \mu \mathrm{m}$ (av. $=12 \times 2 \mu \mathrm{m})$, frequently slightly flattened at the base, held in asymmetrical clusters by colourless slime.

Culture characteristics - Colonies convex, cottony, with smooth margins, cream with pale luteous centre; reverse pale luteous to honey with sepia centre; chlamydospores extensive throughout medium arranged in chains; reaching $50-75 \mathrm{~mm}$ after 7 days on MEA at $24{ }^{\circ} \mathrm{C}$ in ambient light. 


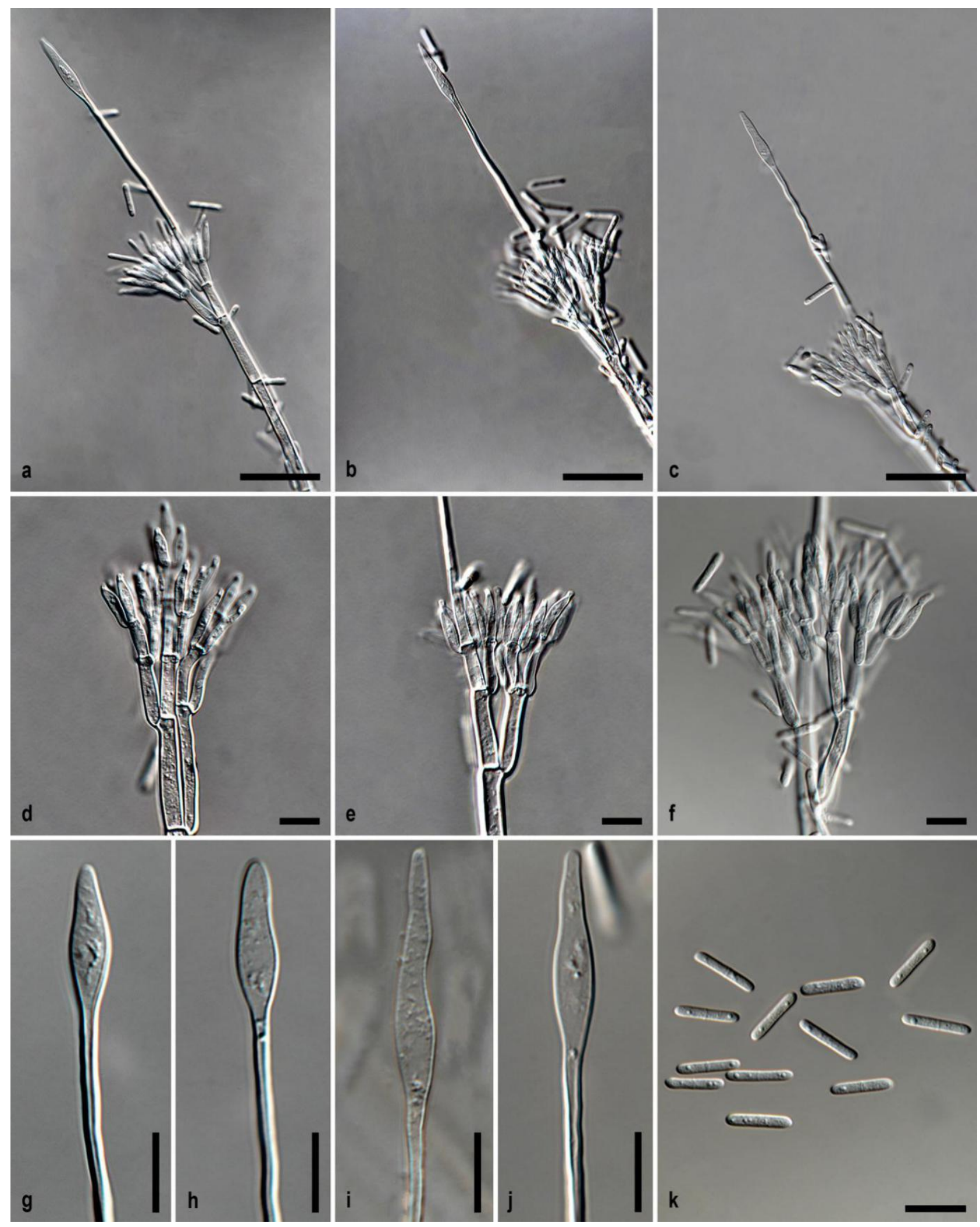

Figure 2 - Cylindrocladiella brevistipitata (ex-type CBS 142786). a-c. Penicillate conidiophores. d-f. Penicillate conidiogenous apparatus. $\mathbf{g}-\mathbf{j}$. Thin-walled, ellipsoidal to lanceolate vesicles. $\mathbf{k}$. Conidia. Scale bars $\mathrm{a}-\mathrm{c}=20 \mu \mathrm{m} ; \mathrm{d}-\mathrm{k}=10 \mu \mathrm{m}$.

Material examined - Thailand, Mae Rim, Queen Sirikit Botanical Garden, from soil, October 2010, P.W. Crous (holotype CBS H-23174, culture ex-type CBS 142784); ibid., culture CBS 142785. 
Notes - Cylindrocladiella horticola displays some morphological overlap with $C$. kurandica, C. pseudoinfestans and C. queenslandica (Schoch et al. 2000, Crous 2002, Lombard et al. 2012). The conidia of $C$. horticola [(11-)11.5-12.5(-14) $\times(1-) 1.5-2.5(-3) \mu \mathrm{m}(\mathrm{av} .=12 \times 2 \mu \mathrm{m})]$ are slightly smaller than those of $C$. kurandica $[(10-) 12-14(-16) \times 2-4) \mu \mathrm{m}(\mathrm{av} .=13 \times 3 \mu \mathrm{m})$; Lombard et al. 2012] and C. pseudoinfestans [(10-)14-16(-20) $\times 2(-3) \mu \mathrm{m}(\mathrm{av} .=15 \times 2 \mu \mathrm{m})$; Schoch et al. 2000 ] but overlap with those of $C$. queenslandica [(9-)10.5-13.5(-15) $\times 2-4 \mu \mathrm{m}(\mathrm{av} .=12 \times 3 \mu \mathrm{m})$; Lombard et al. 2012]. However, the stipe extension of $C$. queenslandica (up to $180 \mu \mathrm{m}$; Lombard et al. 2012) is longer than that of $C$. horticola (up to $150 \mu \mathrm{m}$ ).

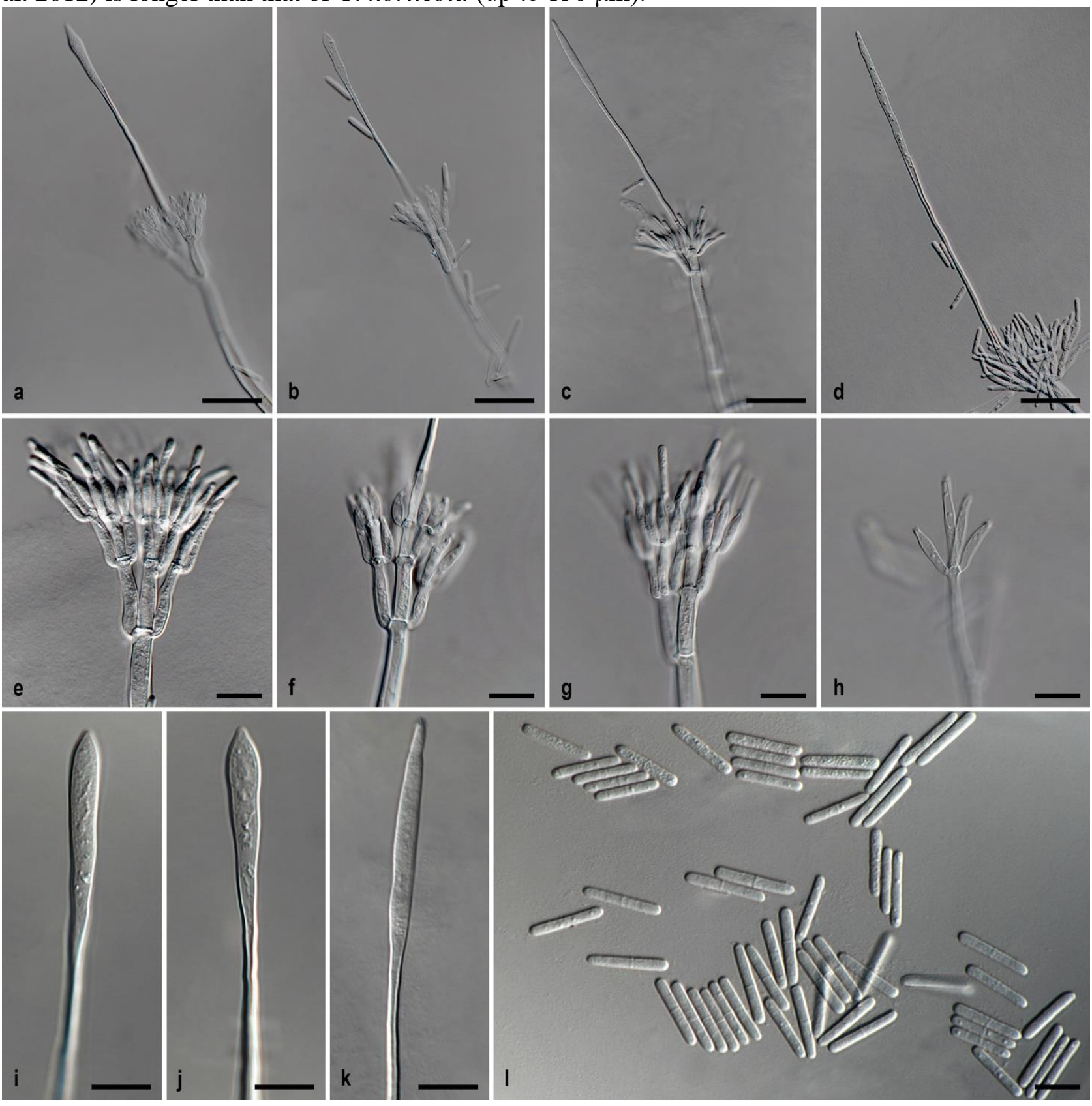

Figure 3 - Cylindrocladiella horticola (ex-type CBS 142784). a-d. Penicillate conidiophores. e-g. Penicillate conidiogenous apparatus. $\mathbf{h}$. Subverticillate conidiophores. $\mathbf{i}-\mathbf{k}$. Thin-walled, ellipsoidal to lanceolate vesicles. I. Conidia. Scale bars $\mathrm{a}-\mathrm{d}=20 \mu \mathrm{m}$; $\mathrm{e}-\mathrm{l}=10 \mu \mathrm{m}$.

Cylindrocladiella humicola L. Lombard \& Crous, sp. nov.

Fig. 4 MycoBank MB821581; Facesoffungi number: FoF03584

Etymology - Named after the substrate, soil, from which the fungus was isolated. 
Sexual morph unknown. Conidiophores penicillate, mononematous and hyaline, comprising a stipe, a penicillate arrangement of fertile branches, a stipe extension and a terminal vesicle; stipe septate, hyaline, smooth, 30-60 × $5 \mu \mathrm{m}$; stipe extension aseptate, straight, $85-110 \mu \mathrm{m}$ long, thickwalled with one basal septum, terminating in thin-walled, elongate clavate vesicles, $2-4 \mu \mathrm{m}$ wide. Penicillate conidiogenous apparatus with primary branches aseptate, 10-20 $\times 2-4 \mu \mathrm{m}$, secondary branches aseptate, 7-10 $\times 2-3 \mu \mathrm{m}$, each terminal branch producing 2-4 phialides; phialides cymbiform to cylindrical to ventricose, hyaline, aseptate, $7-12 \times 2-3 \mu \mathrm{m}$, apex with minute periclinal thickening and collarette. Subverticillate conidiophores not observed. Conidia cylindrical, rounded at both ends, straight, 1-septate, $(10-) 11-13(-14) \times 1-3 \mu \mathrm{m}(\mathrm{av} .=12 \times 2 \mu \mathrm{m})$, frequently slightly flattened at the base, held in asymmetrical clusters by colourless slime.

Culture characteristics - Colonies convex, cottony, with sinuate margins, cream with pale luteous centre; reverse cinnamon to honey with sepia centre; chlamydospores extensive throughout medium, arranged in chains; reaching $45-70 \mathrm{~mm}$ after 7 days on MEA at $24{ }^{\circ} \mathrm{C}$ in ambient light.

Material examined - Thailand, Chiang Rai, from soil, October 2010, P.W. Crous (holotype CBS H-23175, culture ex-type CBS 142779); ibid., cultures CBS 142777 \& CBS 142778.

Notes - Cylindrocladiella humicola is closely related to $C$. clavata (Fig. 1). The conidia of $C$. humicola $[(10-) 11-13(-14) \times 1-3 \mu \mathrm{m}(\mathrm{av} .=12 \times 2 \mu \mathrm{m})]$ are slightly smaller than those of $C$. clavata [(10-)13-15(-16) $\times 2-3 \mu \mathrm{m}(\mathrm{av} .=14 \times 2 \mu \mathrm{m})$; Lombard et al. 2012] and the stipe extensions of $C$. humicola are shorter $(110 \mu \mathrm{m})$ compared to C. clavata (up to $170 \mu \mathrm{m}$; Lombard et al. 2012).

Cylindrocladiella lateralis L. Lombard \& Crous, sp. nov.

Fig. 5

MycoBank MB821582; Facesoffungi number: FoF03585

Etymology - Named refers to the lateral stipe extensions on the penicillate conidiogenous apparatus.

Sexual morph unknown. Conidiophores dimorphic, penicillate and subverticillate, mononematous and hyaline, comprising a stipe, a penicillate arrangement of fertile branches, stipe extensions terminating in vesicles; stipe septate, hyaline, smooth, 30-90 × 3-7 $\mu \mathrm{m}$; stipe extension aseptate, straight, 100-130 $\mu \mathrm{m}$ long, thick-walled with one basal septum, terminating in thin-walled, elongate clavate to cylindrical vesicles, 3-4 $\mu \mathrm{m}$ wide; lateral stipe extensions sparse, 55-95 $\mu \mathrm{m}$ long, thick-walled with one basal septum, terminating in thin-walled, elongate clavate to cylindrical vesicles, 2-3 $\mu \mathrm{m}$ wide. Penicillate conidiogenous apparatus with primary branches aseptate, 12-22 $\times 2-4 \mu \mathrm{m}$, secondary branches aseptate, 9-16 $\times 2-3 \mu \mathrm{m}$, each terminal branch producing 2-4 phialides; phialides doliiform to reniform to cymbiform, hyaline, aseptate, 7-13 $\times 2-3 \mu \mathrm{m}$, apex with minute periclinal thickening and collarette. Subverticillate conidiophores abundant, comprising of a septate stipe and primary branches terminating in 2-4 phialides; primary branches straight, hyaline, 0-1-septate, $12-45 \times 2-4 \mu \mathrm{m}$; phialides cymbiform to cylindrical, hyaline, aseptate, $15-20 \times 2-3$ $\mu \mathrm{m}$, apex with minute periclinal thickening and collarette. Conidia cylindrical, rounded at both ends, straight, 1-septate, $12-14(-15) \times 1-2 \mu \mathrm{m}(\mathrm{av} .=13 \times 2 \mu \mathrm{m})$, frequently slightly flattened at the base, held in asymmetrical clusters by colourless slime.

Culture characteristics - Colonies convex, cottony, with sinuate margins, cream with pale luteous centre; reverse pale luteous to honey with sepia centre; chlamydospores moderate throughout medium arranged in chains; reaching $45-65 \mathrm{~mm}$ after 7 days on MEA at $24{ }^{\circ} \mathrm{C}$ in ambient light.

Material examined - Thailand, Mae Rim, Queen Sirikit Botanical Garden, from soil, October 2010, P.W. Crous (holotype CBS H-23176, culture ex-type CBS 142788); ibid., culture CBS 142787.

Notes - Cylindrocladiella lateralis is the only species known thus far to produce lateral stipe extensions on its penicillate conidiogenous apparatus, thereby distinguishing it from other species in the genus. 


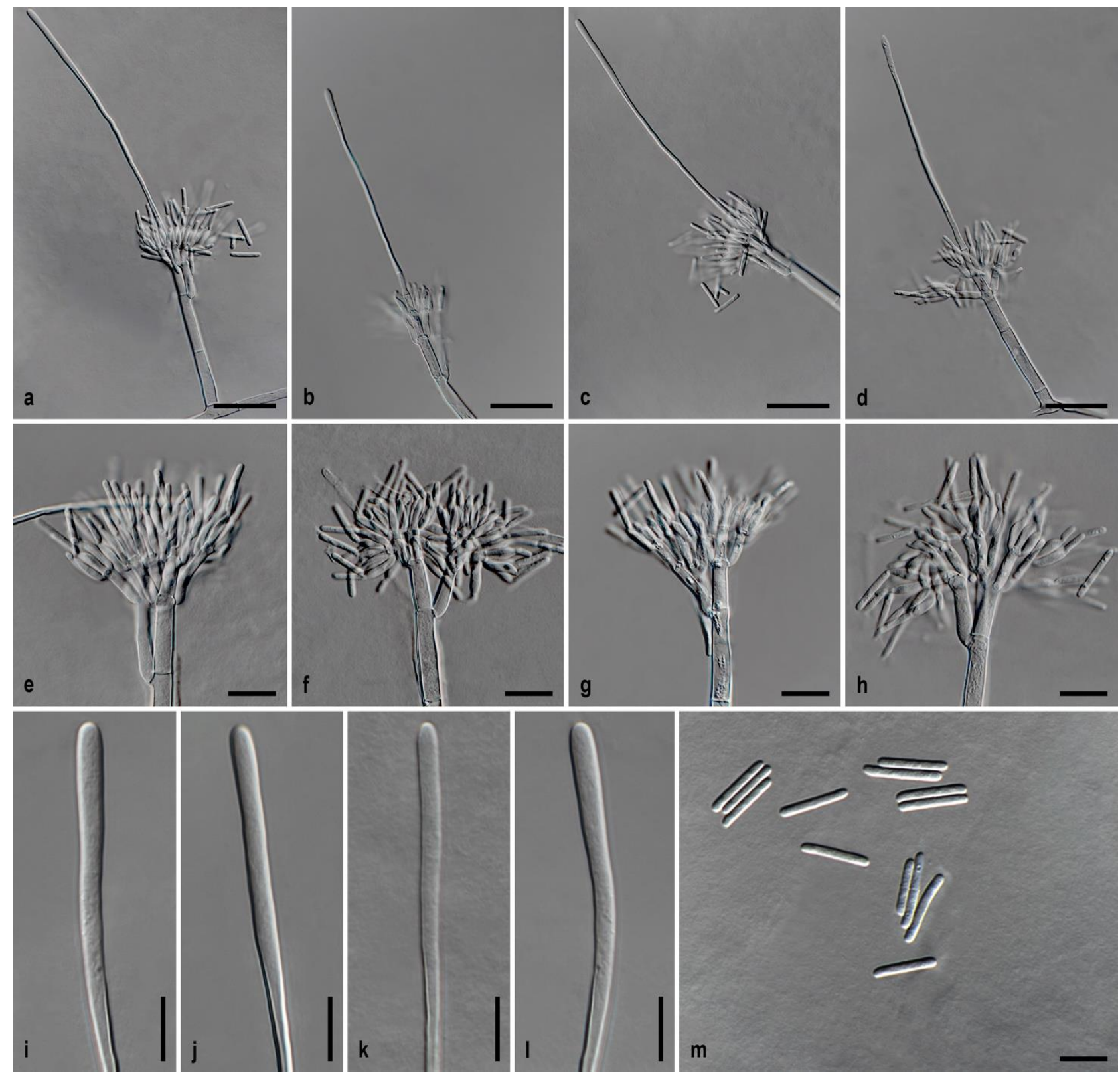

Figure 4 - Cylindrocladiella humicola (ex-type CBS 142779). a-d. Penicillate conidiophores. e-h. Penicillate conidiogenous apparatus. $\mathbf{i}-\mathbf{l}$. Thin-walled, elongate clavate vesicles. m. Conidia. Scale bars $\mathrm{a}-\mathrm{d}=20 \mu \mathrm{m} ; \mathrm{e}-\mathrm{m}=10 \mu \mathrm{m}$.

Cylindrocladiella reginae L. Lombard \& Crous, sp. nov. Fig. 6 MycoBank MB821583; Facesoffungi number: FoF03586

Etymology - Named refers to the locality, Queen Sirikit Botanical Garden, from where this fungus was collected.

Sexual morph unknown. Conidiophores dimorphic, penicillate and subverticillate, mononematous and hyaline, comprising a stipe, a penicillate arrangement of fertile branches, a stipe extension and a terminal vesicle; stipe septate, hyaline, smooth, 40-65 $\times 4-7 \mu \mathrm{m}$; stipe extension aseptate, straight, 85-120 $\mu \mathrm{m}$ long, thick-walled with one basal septum, terminating in thin-walled, elongate clavate vesicles, 3-4 $\mu \mathrm{m}$ wide. Penicillate conidiogenous apparatus with primary branches aseptate, $8-20 \times 2-5 \mu \mathrm{m}$, secondary branches aseptate, $8-13 \times 2-4 \mu \mathrm{m}$, tertiary branches rare, aseptate, $8-10 \times 2-3 \mu \mathrm{m}$, each terminal branch producing 2-4 phialides; phialides cymbiform to cylindrical to ventricose, hyaline, aseptate, $7-12 \times 1-4 \mu \mathrm{m}$, apex with minute periclinal thickening and collarette. Subverticillate conidiophores abundant, comprising of a septate stipe and primary 
branches terminating in 1-3 phialides; primary branches straight, hyaline, $0-1$-septate, $20-45 \times 2-4$ $\mu \mathrm{m}$; phialides cymbiform to cylindrical, hyaline, aseptate, 13-27 $\times 2-3 \mu \mathrm{m}$, apex with minute periclinal thickening and collarette. Conidia cylindrical, rounded at both ends, straight, 1-septate, $(10-) 11.5-12.5(-13) \times 2-3 \mu \mathrm{m}(\mathrm{av} .=12 \times 2 \mu \mathrm{m})$, frequently slightly flattened at the base, held in asymmetrical clusters by colourless slime.

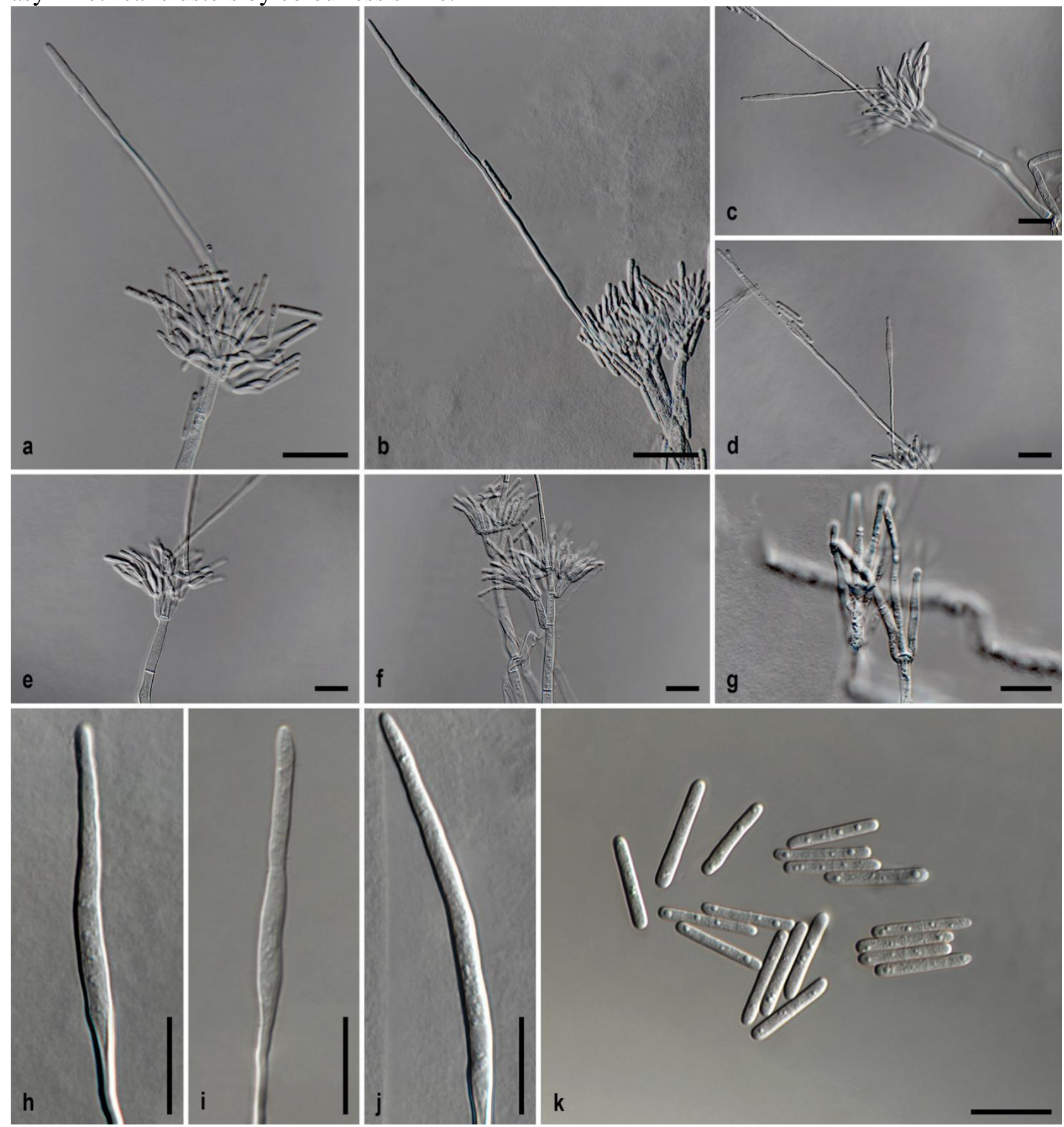

Figure 5 - Cylindrocladiella lateralis (ex-type CBS 142788). a-b. Penicillate conidiophores. c-d. Penicillate conidiophores with lateral stipe extensions. e-f. Penicillate conidiogenous apparatus. g. Subverticillate conidiophores. $\mathbf{h}-\mathbf{j}$. Thin-walled, elongate clavate to cylindrical vesicles. $\mathbf{k}$. Conidia. Scale bars a-d $=20 \mu \mathrm{m}$; $\mathrm{e}-\mathrm{k}=10 \mu \mathrm{m}$.

Culture characteristics - Colonies convex, cottony, with sinuate margins, cream to pale luteous with honey centre; reverse cinnamon to honey with sepia centre and pale luteous margin; chlamydospores extensive throughout medium arranged in chains; reaching 60-65 mm after 7 days on MEA at $24{ }^{\circ} \mathrm{C}$ in ambient light. 
Materials examined - Thailand, Mae Rim, Queen Sirikit Botanical Garden, from soil, October 2010, P.W. Crous (holotype CBS H-23177, culture ex-type CBS 142782); ibid., cultures CBS 142780, CBS 142781 \& CBS 142783.

Notes - Cylindrocladiella reginae can be distinguished from its closest phylogenetic neighbours (C. parva, C. pseudoparva and $C$. stellenboschensis; Fig. 1) by the presence of tertiary branches on its penicillate conidiogenous apparatus, a characteristic not shared by either C. parva, C. pseudoparva and C. stellenboschensis (Boesewinkel 1982, Crous 2002, Lombard et al. 2012).

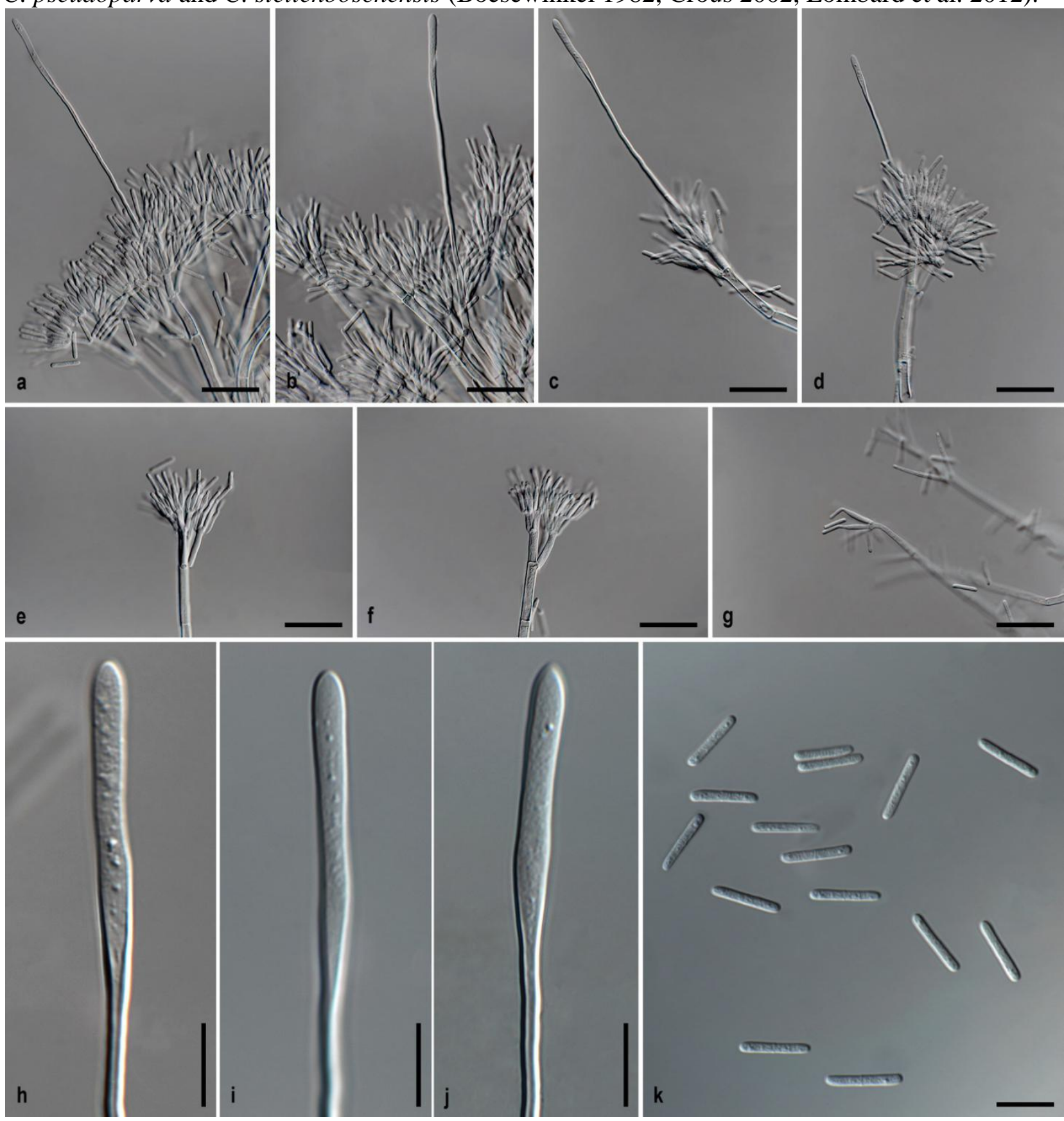

Figure 6 - Cylindrocladiella reginae (ex-type CBS 142782). a-d. Penicillate conidiophores. e-f. Penicillate conidiogenous apparatus. g. Subverticillate conidiophores. $\mathbf{h}-\mathbf{j}$. Thin-walled, elongate clavate vesicles. $\mathbf{k}$. Conidia. Scale bars $\mathrm{a}-\mathrm{d}=20 \mu \mathrm{m} ; \mathrm{e}-\mathrm{k}=10 \mu \mathrm{m}$.

Cylindrocladiella terrestris L. Lombard \& Crous, sp. nov.

Fig. 7

MycoBank MB821584; Facesoffungi number: FoF03587

Etymology - Named after the substrate, soil, from which the fungus was isolated. 


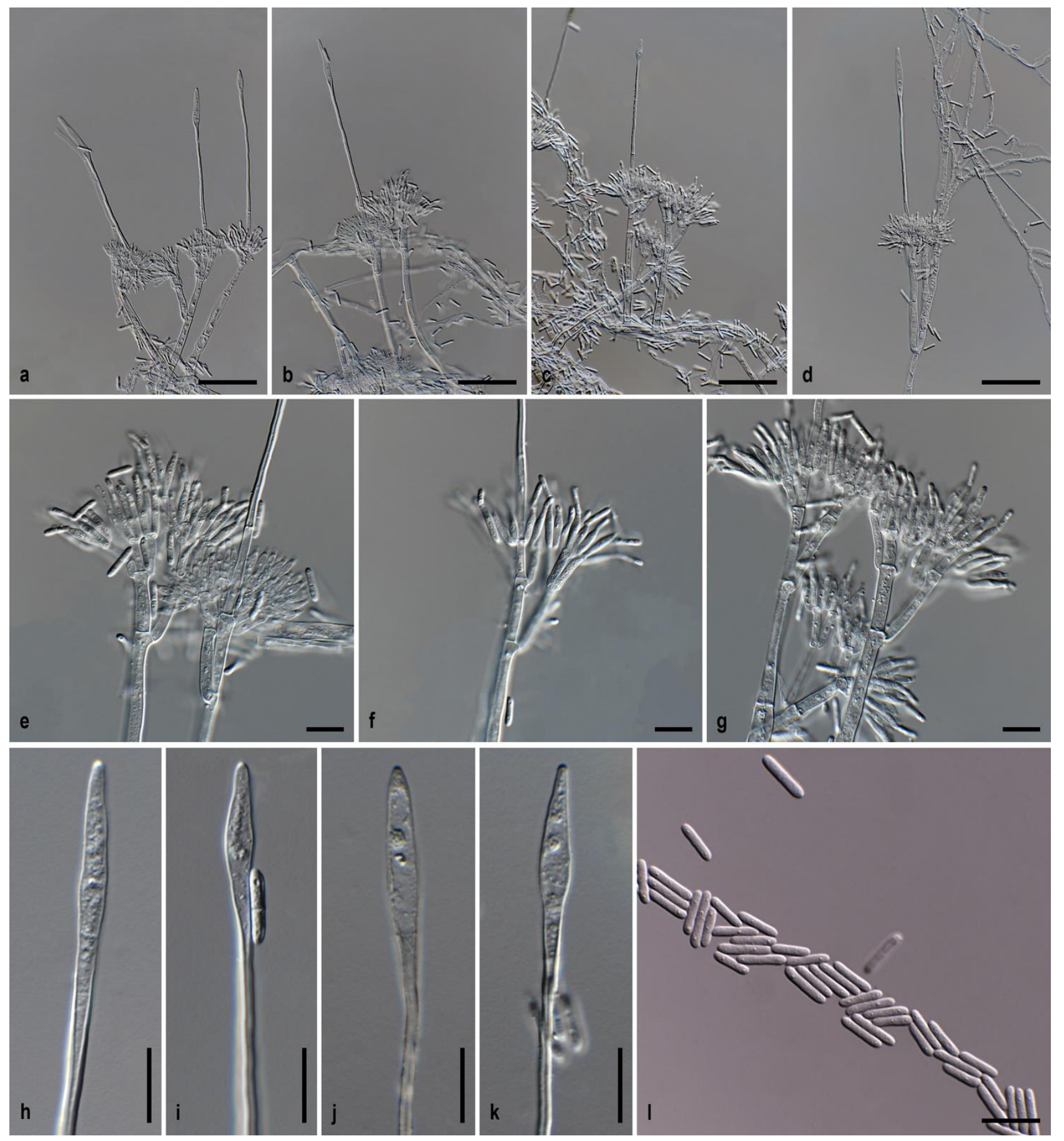

Figure 7 - Cylindrocladiella terrestris (ex-type CBS 142789). a-d. Penicillate conidiophores. e-g. Penicillate conidiogenous apparatus. $\mathbf{h}-\mathbf{k}$. Thin-walled, ellipsoidal to lanceolate vesicles. $\mathbf{I}$. Conidia. Scale bars a $-\mathrm{d}=20 \mu \mathrm{m}$; $\mathrm{e}-1=10 \mu \mathrm{m}$.

Sexual morph unknown. Conidiophores penicillate, mononematous and hyaline, comprising a stipe, a penicillate arrangement of fertile branches, a stipe extension and a terminal vesicle; stipe septate, hyaline, smooth, 50-100 × 6-7 $\mu \mathrm{m}$; stipe extension aseptate, straight, $80-120 \mu \mathrm{m}$ long, thick-walled with one basal septum, terminating in thin-walled, ellipsoidal to lanceolate vesicles, 4(-5) $\mu \mathrm{m}$ wide. Penicillate conidiogenous apparatus with primary branches aseptate, $16-27 \times 3-4$ $\mu \mathrm{m}$, secondary branches aseptate, $10-15 \times 3-4 \mu \mathrm{m}$, each terminal branch producing $2-4$ phialides; phialides cymbiform to cylindrical, hyaline, aseptate, 10-15 $\times 2.5-3.5 \mu \mathrm{m}$, apex with minute periclinal thickening and collarette. Subverticillate conidiophores not observed. Conidia cylindrical, 
rounded at both ends, straight, 1 -septate, $(8-) 10-12(-14) \times 2(-2.5) \mu \mathrm{m}(\mathrm{av} .=11 \times 2 \mu \mathrm{m})$, frequently slightly flattened at the base, held in asymmetrical clusters by colourless slime.

Culture characteristics - Colonies convex, cottony, with sinuate margins, pale luteous with sepia centre; reverse sienna to umber with sepia centre; chlamydospores extensive throughout medium arranged in chains; reaching 60-65 mm after 7 days on MEA at $24{ }^{\circ} \mathrm{C}$ in ambient light.

Material examined - Thailand, Mae Rim, Queen Sirikit Botanical Garden, from soil, October 2010, P.W. Crous (holotype CBS H-23178, culture ex-type CBS 142789); ibid., culture CBS 142790 .

Notes - Cylindrocladiella terrestris is closely related to $C$. peruviana (Fig. 1). The stipe extensions of $C$. terrestris are shorter (up to $120 \mu \mathrm{m}$ long) compared to those of $C$. peruviana (up to $140 \mu \mathrm{m}$ long; Boesewinkel 1982, Crous 2002). Additionally, C. terrestris did not form subverticillate conidiophores in vitro, whereas they are present in C. peruviana (Crous 2002).

\section{Discussion}

The present study introduces six new Cylindrocladiella species, which were all isolated from soil samples collected in northern Thailand. This brings the number of known Cylindrocladiella species from Thailand to 13, and includes C. camelliae, C. longiphialidica, C. microcylindrica, $C$. peruviana, C. pseudocamelliae, C. thailandica and C. viticola (Crous 2002, Lombard et al. 2012). Of these, only $C$. viticola has been isolated from a plant host (Vitis vinifera in South Africa, and Amorphophallus sp. in Thailand; Crous 2002), with the remaining 12 originating from soil (Crous 2002, Lombard et al. 2012). However, although their ecology is presently unknown, their relevance as plant pathogens still needs to be determined as Cylindrocladiella species are generally regarded as weak plant pathogens, especially in nurseries under tropical conditions (Lombard et al. 2012).

Interestingly, the majority of the Cylindrocladiella species presently known from Thailand have thus far been collected only from northern Thailand (Crous 2002, Lombard et al. 2012). The only exceptions are $C$. viticola and $C$. microcylindrica, for which the collection localities within Thailand remains unknown. The apparent high level of species diversity therefore suggests that a more comprehensive soil survey from other regions of Thailand would probably provide a better understanding of the diversity of Cylindrocladiella spp. present in this country. It is hoped that future surveys would be able to elucidate this aspect in more detail.

\section{Acknowledgement}

This work was made possible via an adjunct professorial appointment from the Chiang Mai University to P.W. Crous.

\section{References}

Boesewinkel HJ. 1982 - Cylindrocladiella, a new genus to accommodate Cylindrocladium parvum and other small-spored species of Cylindrocladium. Canadian Journal of Botany 60, 22882294.

Coller GJ Van, Denman S, Groenewald JZ, Lamprecht SC, Crous PW. 2005 - Characterisation and pathogenicity of Cylindrocladiella spp. associated with root and cutting rot symptoms of grapevines in nurseries. Australasian Plant Pathology 34, 489-498.

Crous PW. 2002 - Taxonomy and pathology of Cylindrocladium (Calonectria) and allied genera. APS Press, St. Paul, Minnesota, USA.

Crous PW, Gams W, Stalpers JA, Robert V, Stegehuis G. 2004 - MycoBank: an online initiative to launch mycology into the 21st century. Studies in Mycology 50, 19-22.

Crous PW, Phillips AJL, Wingfield MJ. 1991 - The genera Cylindrocladium and Cylindrocladiella in South Africa, with special reference to forest nurseries. South African Journal of Forestry 157, 69-85. 
Crous PW, Wingfield MJ. 1993 - A re-evaluation of Cylindrocladiella, and a comparison with morphologically similar genera. Mycological Research 97, 433-448.

Crous PW, Wingfield MJ, Burgess TI, Hardy GEStJ et al.. 2017 - Fungal Planet description sheets: 558-624. Persoonia 38, 240-384.

Cunningham CW. 1997 - Can three incongruence tests predict when data should be combined? Molecular Biology and Evolution 14, 733-740.

Gueidan C, Roux C, Lutzoni F. 2007 - Using multigene phylogeny analysis to assess generic delineation and character evolution in Verrucariaceae (Verrucariales, Ascomycota). Mycological Research 111, 1145-1168.

Hillis DM, Bull JJ. 1993 - An empirical test of bootstrapping as a method for assessing confidence in phylogenetic analysis. Systematic Biology 42, 182-192.

Inderbitzin P, Bostock RM, Subbarao KV. 2012 - Cylindrocladiella hahajimaensis, a new species of Cylindrocladiella transferred from Verticillium. MycoKeys 4, 1-8.

Katoh K, Standley DM. 2013 - MAFFT multiple sequence alignment software version 7: Improvements in performance and usability. Molecular Biology and Evolution 30, 772-780.

Lombard L, Shivas RG, To-Anun C, Crous PW. 2012 - Phylogeny and taxonomy of the genus Cylindrocladiella. Mycological Progress 11, 835-868.

Lombard L, van der Merwe NA, Groenewald JZ, Crous PW. 2015 - Generic concepts in Nectriaceae. Studies in Mycology 80, 189-245.

McNiell J, Barrie FF, Buck WR, Demoulin V et al. (eds.) 2012 - International Code of Nomenclature for algae, fungi and plants (Melbourne Code). [Regnum Vegetabile no. 154] A.R.G. Gantner Verlag KG.

Nirenberg HI. 1981 - A simplified method for identifying Fusarium spp. occurring on wheat. Canadian Journal of Botany 59, 1599-1609.

Nylander JAA. 2004 - MrModeltest v. 2. Programme distributed by the author. Uppsala: Evolutionary Biology Centre, Uppsala University.

Peerally A. 1991 - The classification and phytopathology of Cylindrocladium species. Mycotaxon 40, 323-366.

Rayner RW. 1970 - A Mycological Colour Chart. Kew: Commonwealth Mycological Institute.

Ronquist F, Huelsenbeck JP. 2003 - MrBayes 3: Bayesian phylogenetic inference under mixed models. Bioinformatics 19, 1572-1574.

Rossman AY, Seifert KA, Samuels GJ, Minnis AM et al. 2013 - Genera in Bionectriaceae, Hypocreaceae, and Nectriaceae (Hypocreales) proposed for acceptance or rejection. IMA Fungus 4, 41-51.

Scattolin L, Montecchio L. 2007 - First report of damping-off of common oak plantlets caused by Cylindrocladiella parva in Italy. Plant Disease 91, 771.

Schoch CL, Crous PW, Wingfield MJ, Wingfield BD. 2000 - Phylogeny of Calonectria and selected hypocrealean genera with cylindrical macroconidia. Studies in Mycology 45, 45-62.

Stamatakis A. 2014 - RAxML Version 8: A tool for phylogenetic analysis and post-analysis of large phylogenies. Bioinformatics 30, 1312-1313.

Swofford DL. 2003 - PAUP*: phylogenetic analysis using parsimony (*and other methods). Version 4.0b10. Sunderland, MA: Sinauer Associates. 